Proceedings of the 37th Polish Seminar on Positron Annihilation, Lądek-Zdrój 2007

\title{
Nanopores in Silica Aerogels
}

\author{
O. ŠAuŠA ${ }^{a}$, M. Iskrováa $a$, V. MAJerníK ${ }^{a}$, J. KRIŠtiaK $^{a}$, \\ K. JESEnÁK ${ }^{b}$ AND M. VARGOVÁ ${ }^{b}$ \\ ${ }^{a}$ Institute of Physics of SAS, Dúbravská cesta 9, 85411 Bratislava, Slovakia \\ ${ }^{b}$ Department of Inorganic Chemistry, Faculty of Natural Sciences \\ Comenius University in Bratislava \\ Mlynská Dolina CH-2, 84215 Bratislava, Slovakia
}

\begin{abstract}
The size of nanopores in thermally treated samples of silica aerogels was determined by ortho-positronium lifetime measurements at room temperature. The annealing of samples in the temperature region of $300-800^{\circ} \mathrm{C}$ does not make substantial changes in pore size felt by positronium. The annealing at higher temperatures from an interval of $800-1050^{\circ} \mathrm{C}$ causes shrinkage of the samples reducing the pore size to $1-15 \mathrm{~nm}$. The macropores vanish at temperature treatment at $1000^{\circ} \mathrm{C}$ and the positron annihilation is not affected by oxygen from air.

PACS numbers: 78.70.Bj
\end{abstract}

\section{Introduction}

The size and density of pores in silica aerogels depends on technological processes of their preparation. There are several standard techniques for pore size determination and positron annihilation lifetime spectroscopy (PALS) method belongs to them.

PALS is based on lifetime measurements of positron or positronium (Ps) in analyzed surroundings. The vacuum lifetime of the ortho-Ps (o-Ps) (142 ns) is reduced because $o$-Ps can pick off an electron of opposite spin from surroundings. The lifetime reduction depends markedly on the average electron density in the walls of pores and thus information on their sizes can be deduced from the o-Ps lifetimes.

The quantum mechanical model for the probability of o-Ps annihilation trapped in small pores was elaborated by different authors [1-3]. It consists of a spherical potential well, in which the single particle states of Ps atom are calculated. Only the ground state in the well is considered and the pick-off annihilation depends both on the overlap of the positron from o-Ps atom and the molecular-electron wave function.

This standard scheme is certainly an approximation because the positronium as an inserted probe detects the empty space as many isolated irregular shaped, local free volumes of atomic and molecular dimension. 
At cases of very long lifetimes, for example in the case of high porosity silica aerogels with very large cavities about ten of $\mathrm{nm}$, this standard model is not valid. The average $o$-Ps lifetimes are within a few percent of vacuum value of $142 \mathrm{~ns}$ and sensitivity of PALS method for determination of pore size larger than $20 \mathrm{~nm}$ goes down. Progress has been made in the development of different models for the extension of standard Tao-Eldrup formula $[1,2]$ to cavities larger than $1 \mathrm{~nm}$ in radius [4-6]. Moreover, for such large cavities significant fractions of the Ps atoms will be in excited states of the potential well due to high density of states. The calculations of the Ps lifetime based on this physical idea were proposed in several papers $[7,8]$.

There is a problem connected with geometry of pores. There are different approaches for solving of the $o$-Ps lifetime dependence on the shape of cavities (sphere, cylinder, cube, etc.) [7-11]. In this work, the o-Ps lifetimes measured in silica aerogels have been converted to pore dimensions using the calculations reported by $[7,12]$.

\section{Experimental}

The $\mathrm{SiO}_{2}$ aerogel was produced by supercritical drying of alcogel $(T=$ $270^{\circ} \mathrm{C}, p=12 \mathrm{MPa}$ ) through hydrolysis of the tetramethoxysilane solution in methyl alcohol in a weakly alkaline ammonia medium followed by exposure to methyl alcohol for 7 days under normal conditions. The volume ratio of tetramethoxysilane, methyl alcohol and water in the initial solution was $2: 2: 1$. Then the $\mathrm{SiO}_{2}$ aerogel was annealed at $300^{\circ} \mathrm{C}$ for $4 \mathrm{~h}$. The density and refractive index of the finished samples was $\rho=0.18 \mathrm{~g} / \mathrm{cm}^{3}$ and $n=1.038$, respectively [13].

In next steps the samples of this initial material was heated for $3 \mathrm{~h}$ at chosen temperature. Then annihilation spectra were measured at $300 \mathrm{~K}$ in air and in vacuum. We used bulk (monolithic) or granulated samples with average size of grains $0.2-0.5 \mathrm{~mm}$.

Two disc shaped containers filled with specific silica gel samples were placed on either side of Na-22 positron source. This was $2 \mathrm{MBq}$ activity spot sandwiched between $8 \mu \mathrm{m}$ kapton foils.

The positron annihilation lifetime spectra were obtained by conventional fast-fast coincidence method using plastic scintillators coupled to Phillips XP2020 photomultipliers. The time resolution (FWHM) was about $350 \mathrm{ps}$. Total number of coincidences in analyzed time spectra exceeds $1 \times 10^{6}$ counts.

A standard four component analysis of lifetime spectra was carried out using the LT program [14]. Source correction were taken into account.

\section{Results and discussion}

The average size of micropores and mesopores of the initial material was deduced from the 4-component analysis of lifetime spectra at room temperature and vacuum using lifetime-pore size conversion tables [12]. The values are presented 
in Table. The good approximation of the free volume in the net structure of silica aerogels is the spheric shaped pore with radius $R_{\mathrm{s}}$, eventually, we can use the approximation by long cylinder with radius $R_{\mathrm{c}}$ of the pedestal in case of free volumes connected to channel in which $o$-Ps freely diffuses. The value $\Delta=0.19 \mathrm{~nm}$ provides good correspondence of the theoretical calculations for cylindrical pore with the experimental values $\tau_{o-P s}$ of the well-known pore sizes materials [15]. Therefore, this value of $\Delta$ was used in our work for the estimation pore sizes.

TABLE

$o$-Ps lifetime, intensity and estimated radius of large pores of the primary monolithic sample. Silica aerogel, measurements at $300 \mathrm{~K}$ in vacuum. The conversion of the $o$-Ps lifetime to radius of pores estimated by [12]. $R_{\mathrm{s}}-$ radius of spherical shaped pores, $R_{\mathrm{c}}$ - radius of pedestal of cylindrical shaped pores (both in $[\mathrm{nm}]$ ).

\begin{tabular}{c|c|c|c|c|c|c|c|c}
\hline \hline$\tau_{3}[\mathrm{~ns}]$ & $I_{3}[\%]$ & $\tau_{4}[\mathrm{~ns}]$ & $I_{4}[\%]$ & $R_{\mathrm{s} 3}$ & $R_{\mathrm{c} 3}$ & $R_{\mathrm{s} 4}$ & $R_{\mathrm{c} 4}$ & $\Delta[\mathrm{nm}]$ \\
\hline $3.6(0.3)$ & $1.4(0.1)$ & $114.4(1.6)$ & $12.6(0.2)$ & 0.44 & 0.37 & 7.5 & 7.5 & 0.19
\end{tabular}

From the PALS measurements we can see that the mesopores felt by positronium are dominant (90\%) and their average size (diameter) is about $15 \mathrm{~nm}$. The independent information of size of pores was obtained using scanning electron microscopy. The photo of the initial silica aerogel sample surface from the scanning electron microscope shows the typical pores (Fig. 1).

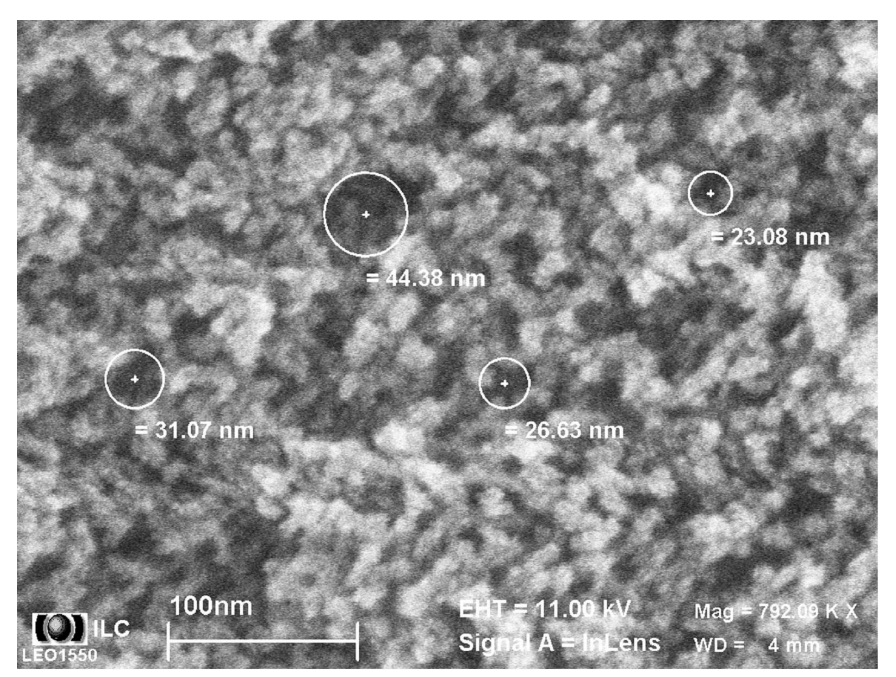

Fig. 1. Picture of silica aerogel basic sample surface from scaning electron microscope. Scale $100 \mathrm{~nm}$. Some mesopores with displayed size. 


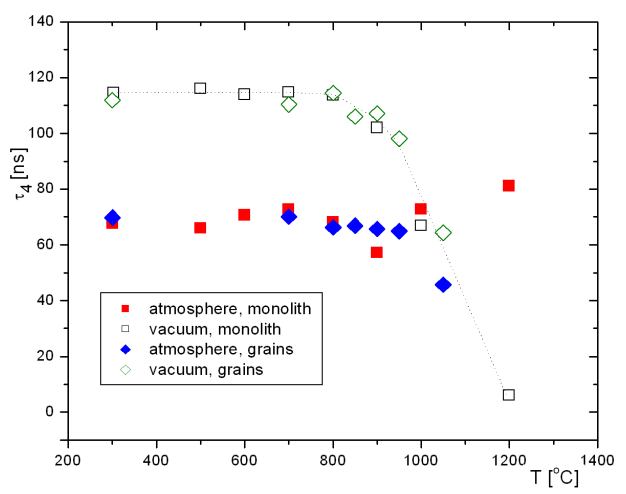

Fig. 2. o-Ps lifetime dependence on annealing temperature $T$. Measurements in air and vacuum at room temperature for different samples (monolith, granules).

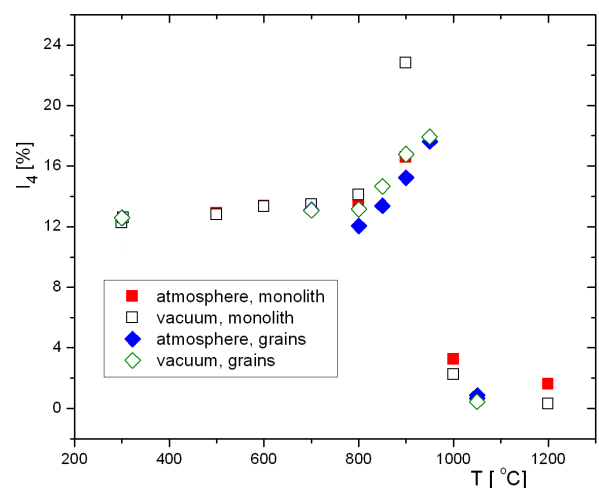

Fig. 3. $o$-Ps lifetime intensity $I_{4}$ dependence on annealing temperature. Measurements in air and vacuum at room temperature for different samples (monolith, granules).

The $o$-Ps lifetime and their intensity dependence on the heat treatment temperature measured in vacuum and air at $300 \mathrm{~K}$ are in Figs. 2 and 3, respectively. We can see that these annihilation parameters are independent of the sample form (monolith, granules).

The heat treatment induces changes in the lifetimes and their intensities at temperature above $800^{\circ} \mathrm{C}$ (Fig. 2). The pore size of mesopores "felt" by $o$-Ps is shrinking to temperature $1200^{\circ} \mathrm{C}$, at which the bigger pores vanish and the sample obtains the glass like structure with pore size diameter about $1.1 \mathrm{~nm}$. The further increase in treatment temperature and variation of time of treatment give the crystalline silica structure [16]. We observe the growth of the intensity $I_{4}$ in the temperature region $800-950^{\circ} \mathrm{C}$ (Fig. 3). Above this temperature the annihilation of $o$-Ps in mesopores rapidly decreases. The $o$-Ps annihilation moves to micropores, because mesopores vanish ( $I_{3}$ increases). The air presence affects the lifetime in big pores. We do not observe this influence in small pores (no 


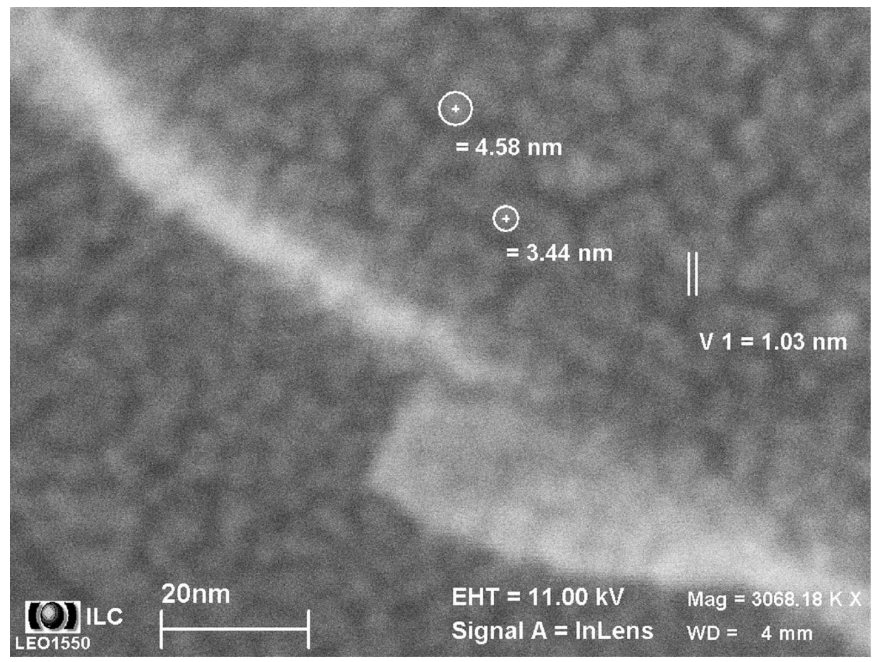

Fig. 4. Picture of silica aerogel sample surface from scanning electron microscope. Scale $20 \mathrm{~nm}$. Some typical structure with displayed size for annealing temperature $1200^{\circ} \mathrm{C}$.

change in $\tau_{3}$ ). The photo of the silica aerogel surface from the scanning electron microscope in Fig. 4 shows structure at heat treatment temperature $1200^{\circ} \mathrm{C}$. The $o$-Ps lifetime reduction in temperature region $800-1050^{\circ} \mathrm{C}$ is similar to observed linear shrinkage of macroscopic size of our samples. Such macroscopic shrinkage was reported in [17], too.

\section{Conclusions}

The influence of heat treatment of silica aerogel samples (the size and nature of pores) by PALS method was studied in this work. The main results can be summarized in the following way:

- Heat treatment at temperature range of $300-800^{\circ} \mathrm{C}$ does not change pore size felt by positrons.

- The heat treatment of samples at the temperature interval $800-1050^{\circ} \mathrm{C}$ affects the average pore size in the region $15-1 \mathrm{~nm}$.

- The large pores vanish at the temperature about $1000^{\circ} \mathrm{C}$.

- Small pores are closed for some gases (minimal influence of oxygen and desorption of gases for samples treated above $950^{\circ} \mathrm{C}$ ).

- The comparison of PALS parameters for the samples with different morphology (monolith, powders, granules) shows that there are no essential differencies between them in lifetime of $o$-Ps, which reflect the pore size.

The positron annihilation method allows to observe the pore size and their nature also in the region, in which other methods show deficiency because of closed pores or limited pore size (nitrogen sorption, mercury porosimetry). The 
PALS measurements are important for the determination of pore size and with combination of an energy spectra analysis (Doppler broadened annihilation line, three gamma annihilation spectra) will be possible to obtain more complex picture about the nature of pores in this very promising material.

\section{Acknowledgments}

The authors O.Š., J.K., M.I. and V.M. wish to thank the grant agency VEGA Slovakia for support by the grants no $2 / 7121 / 27$.

The authors also wish to thank gratefully to Prof. A. Šatka from International Laser Centre Bratislava for the pictures from the FEG scanning electron microscope.

\section{References}

[1] S.J. Tao, J. Chem. Phys. 56, 5499 (1972).

[2] M. Eldrup, D. Lightbody, J.N. Sherwood, Chem. Phys. 63, 51 (1981).

[3] H. Nakanishi, Y.C. Jean, Positron and Positronium Chemistry, Elsevier, Amsterdam 1988

[4] K. Ito, H. Nakanishi, Y. Ujihira, J. Phys. Chem. B 103, 4555 (1999).

[5] T.L. Dull, W.E. Frieze, D.W. Gidley, J.N. Sun, A.F. Yee, J. Phys. Chem. B 105, 4657 (2001).

[6] D.W. Gidley, K.A. Marko, A. Rich, Phys. Rev. Lett. 36, 395 (1976).

[7] T. Goworek, K. Ciesielski, B. Jasinska, J. Wawryszczuk, Chem. Phys. Lett. 272, 91 (1997).

[8] D.W. Gidley, W.E. Frieze, T.L. Dull, A.F. Yee, E.T. Ryan, H.M. Ho, Phys. Rev. B 60, R5157 (1999).

[9] Y.C. Jean, H. Shi, J. Non-Cryst. Solids 172-174, 806 (1994).

[10] G. Consolati, J. Chem. Phys. 117, 7279 (2002).

[11] M.V. Félix, R.A. Rodríguez-Rojas, J. Castaneda-Contreras, R. Morones, V.M. Castano, Opt. Mater. 27, 1291 (2005).

[12] R. Zaleski, Positronium Lifetime vs. Temperature and Free Volume Size Tables. Pick-Off Model Calculations, Institute of Physics, Maria Curie-Skłodowska University, Lublin 2002.

[13] I.R. Boyko, M.A. Ignatenko, K. Esenak, L. Kuchta, J. Ružička, V. Fainor, Nucl. Instrum. Methods Phys. Res. A 363, 568 (1995).

[14] J. Kansy, Nucl. Instr. Methods Phys. Res. A 374, 235 (1996).

[15] K. Cieselski, A.L. Dawidowicz, T. Goworek, B. Jasinska, J. Wawryszczuk, Chem. Phys. Lett. 289, 41 (1998).

[16] C. Hugenschmidt, U. Holzwarth, M. Jansen, S. Kohn, K. Maier, J. Non-Cryst. Solids 217, 72 (1997).

[17] B. Himmel, Th. Gerber, H. Burger, G. Holzhutter, A. Olbertz, J. Non-Cryst. Solids 186, 149 (1995). 